\title{
Non-Cancer Diagnosis
}

National Cancer Institute

\section{Source}

National Cancer Institute. Non-Cancer Diagnosis. NCI Thesaurus. Code C115274.

A term that refers to a broad group of diagnoses that may include any disease except for cancer or cancer related disorders. 